\title{
Awake Prone Positioning in the Management of COVID-19 Pneumonia: A Systematic Review
}

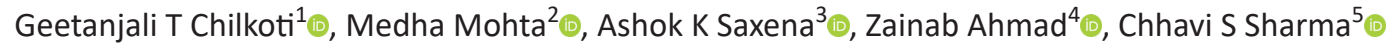

\begin{abstract}
Background: The aim was to investigate the efficacy of prone positioning (PP) in the management of coronavirus disease-2019 (COVID-19) pneumonia in various setups, with various modes of oxygen therapy and its optimal duration.

Materials and methods: A systematic literature search was conducted from inception until May 15, 2021. Patients with a validated diagnosis of COVID-19 and receiving PP were included. Various factors, including intensive care unit (ICU) or non-ICU setup, mode of oxygen therapy, outcome, duration of proning, and limitations, were noted.

Results: We retrieved 36 articles with a total of 1,385 patients for qualitative analysis. Out of 36 articles, there were 17 original articles, 09 case series, and 10 case reports. Out of 1,385 participants, $78.9 \%(n=1,093)$ and $21.0 \%(n=292)$ of patients were managed in ICU and non-ICU setup, respectively. Awake PP with high flow nasal cannula (HFNC) was found to be a promising technique; however, the result was inconclusive with helmet continuous positive airway pressure (CPAP). No study has evaluated the optimal duration of awake PP and the associated long-term outcomes.

Conclusion: We encourage the use of early awake self-proning in the management of COVID19 disease. However, the evidence in terms of its use in non-ICU setup, the optimal duration of PP, and various oxygenation devices are insufficient, thereby mandating further well-designed multicentric studies to evaluate its efficacy as an adjunct in the management of COVID-19 pneumonia in context to the aforementioned factor. Keywords: COVID-19 pneumonia, Management, Prone positioning.

Indian Journal of Critical Care Medicine (2021): 10.5005/jp-journals-10071-23932
\end{abstract}

\section{INTRODUCTION}

Prone positioning (PP) has been an established technique for improving oxygenation in severe acute respiratory distress syndrome (ARDS). ${ }^{1-3}$

Considering the proven benefits of PP in intubated patients, physiologically, it was also assumed to benefit awake, nonintubated patients with acute hypoxemic respiratory failure. With the recent coronavirus disease-2019 (COVID-19) surge, awake self-PP has been practiced widely in the treatment of moderate to severe acute respiratory syndrome coronavirus 2 (SARS-CoV-2) infection. It is a low-risk intervention requiring minimal assistance and, therefore, has also been applied outside the intensive care unit (ICU). Recently, the UK Intensive Care Society has also advocated awake PP as a standard of care for suspected or confirmed COVID-19 patients requiring a $\mathrm{FiO}_{2} \geq 28 \%$. ${ }^{4}$ However, Chad et al. in a review implicated this short-term improvement in oxygenation with PP to be simply a "recruitment maneuver," which could have been efficacious in only patients with less severe disease. ${ }^{5}$ In addition, the patient population assessed in various studies evaluating awake PP in SARS-CoV-2 is heterogeneous in terms of the severity of illness, mode of oxygen therapy, ventilatory status, treatment protocol, mean duration of proning, and the setting, that is, ICU or non-ICU.

As COVID-19 is a novel viral disease, and the evidence available so far to support the efficacy of awake PP is limited; this systematic review was conducted to investigate its efficacy in both awake and intubated patients as an adjunct along with different modes of oxygen therapy or respiratory support, its performance in different setups, that is, ICU or non-ICU, and also the optimal duration of PP.
${ }^{1-5}$ Department of Anaesthesiology, University College of Medical Sciences, New Delhi, India

Corresponding Author: Geetanjali T Chilkoti, Department of Anaesthesiology, University College of Medical Sciences, New Delhi, India, Phone: +91 09711210772, e-mail: geetanjalidr@yahoo.co.in

How to cite this article: Chilkoti GT, Mohta M, Saxena AK, Ahmad $Z$, Sharma CS. Awake Prone Positioning in the Management of COVID-19 Pneumonia: A Systematic Review. Indian J Crit Care Med 2021;25(8):896-905.

Source of support: Nil

Conflict of interest: None

\section{Literature Search and Data Source}

We conducted a comprehensive literature search using PubMed, MEDLINE, Embase, and Google Scholar from December 2019 to May 15, 2020. In PubMed, the following search strategy was used: "(COVID-19 OR Novel Coronavirus-Infected Pneumonia OR 2019 novel coronavirus OR SARS-CoV-2) AND (prone oxygenation OR awake prone position OR self proning)." The strategy was then further adapted for other databases. The titles and abstracts were reviewed to evaluate their relevance to our study. Full-text articles were retrieved for further consideration for inclusion. Two authors (G.T.C. and M.M.) read all the articles, and any inconsistencies were resolved by consensus with the third author (A.K.S.).

Study Selection-For study selection, we followed PICO framework: participants, who had a validated diagnosis of COVID-19, irrespective of stage or severity of disease; intervention, oxygen therapy or respiratory support in awake self-PP; comparison, patients not receiving prone oxygenation, 
if original article; outcomes, various parameters indicating oxygenation. A priori, both interventional and observational data were considered.

Data Extraction-No language restriction was imposed, in order to include maximum articles and minimize language bias. For each article, we extracted data regarding authors, year of publication, the period of observation, patient selection, ICU or non-ICU setting, duration of PP, outcomes assessed, conclusion, and limitations, if any. For the present systematic review, due to the novel nature of the disease, all kinds of publications, that is, case report, case series, editorials, letters, and reviews in addition to original articles providing evidence toward the efficacy of awake PP in the improvement of oxygenation in COVID-19 disease, were included.

\section{Results}

Flowchart 1 shows the PRISMA flowchart depicting the qualitative synthesis of evidence from the literature search. Following the screening of titles, abstracts, and removal of duplicates, finally, we included 36 articles with a total of 1,385 patients for qualitative analysis. Out of 36,17 were original articles, ${ }^{6-22}$ nine case series, $^{23-31}$ and 10 case reports. ${ }^{32-41}$ In addition, there were seven protocols, ${ }^{42-48}$ seven reviews, ${ }^{49-55}$ two commentaries, ${ }^{21,56}$ and four editorial. ${ }^{57-60}$ The 17 original articles were included in the qualitative assessment of risk of bias. All the included articles were published from the inception of COVID-19 till May 15, 2021.

Table 1 shows the characteristics of all the clinical studies evaluating PP in COVID-19 pneumonia.

\section{Awake PP in COVID-19 Pneumonia in Non-ICU Setup}

Out of all the articles, six studies have evaluated awake PP used outside the ICU for COVID-19 pneumonia. 6,7,10,14,19,22 Caputo et al. applied PP to 50 COVID-19 patients in the Emergency Department and showed a significant improvement in oxygenation. ${ }^{6}$ A one-day cross-sectional, before-after study was conducted by Sartini et al. on 15 awake non-ICU patients on noninvasive ventilation (NIV) irrespective of the day. They recorded $\mathrm{SpO}_{2}, \mathrm{PaO}_{2} / \mathrm{FiO}_{2}$, respiratory rate $(\mathrm{RR})$, and patients' comfort at three designated time points while receiving NIV in PP, that is, before starting NIV, 60 minutes after the start of PP, and 60 minutes after the end of NIV. A significant improvement in $\mathrm{SpO}_{2}$ and $\mathrm{PaO}_{2} / \mathrm{FiO}_{2}$ from 100 (IQR, 60-112) to 122 (IQR, 118-122) ( $p<0.001$ for both) along with a decrease in RR during NIV in PP was observed. On follow-up at day 14 , nine patients were discharged, one improved, one was intubated, and one died. ${ }^{10}$

On the contrary, Elharrar et al. in a single-center, before-after study, in patients receiving NIV, observed that out of $24, \mathrm{PaO}_{2}$ improved in only six patients, that is, merely $25 \%$ with PP, whereas four patients did not tolerate PP for more than an hour and required intubation. ${ }^{7}$

\section{Awake PP in COVID-19 Pneumonia in ICU Setup}

Out of all the studies conducted in ICU, only Zang et al. ${ }^{13}$ had incorporated a control group and compared the oxygenation status of patients receiving PP with the ones who did not receive it. The oxygenation parameters used were $\mathrm{SpO}_{2}, \mathrm{RR}$, and ROX index. They did not compare the $\mathrm{PaO}_{2} / \mathrm{FiO}_{2}$ ratio, and also the number of patients with severe diseases was limited in their study.

In another study, Tu et al. exclusively enrolled nine patients with COVID-19 on flow nasal cannula (HFNC) for more than 2 days and having $\mathrm{PaO}_{2} / \mathrm{FiO}_{2}<150 \mathrm{~mm} \mathrm{Hg}$. Prone position was found to be efficacious in improving oxygenation in patients on HFNC. ${ }^{8}$

Similarly, Coppo et al., ${ }^{9}$ in a prospective cohort study, assessed the feasibility of PP in 56 patients receiving NIV or conventional oxygen therapy (COT) and found it to be feasible in $83.9 \%$ of patients $(n=47)$. Oxygenation improved $\mathrm{PaO}_{2} / \mathrm{FiO}_{2}$ ratio from 180.5 to $285.5 \mathrm{~mm} \mathrm{Hg}(112.9)$ in PP ( $p<0.0001)$. Oxygenation following resupination was maintained in only 23 patients (50\%). It was concluded that PP was feasible and effective in improving oxygenation in awake patients with COVID-19; however, the effect was sustained in only $50 \%$ of patients.

Thompson et al. ${ }^{11}$ in a cohort study on 25 patients also observed the efficacy of PP in improving the oxygenation and its effects on intubation rate. They observed that 1 hour after

Flowchart 1: PRISMA flowchart depicting the steps of qualitative synthesis of evidence from the literature

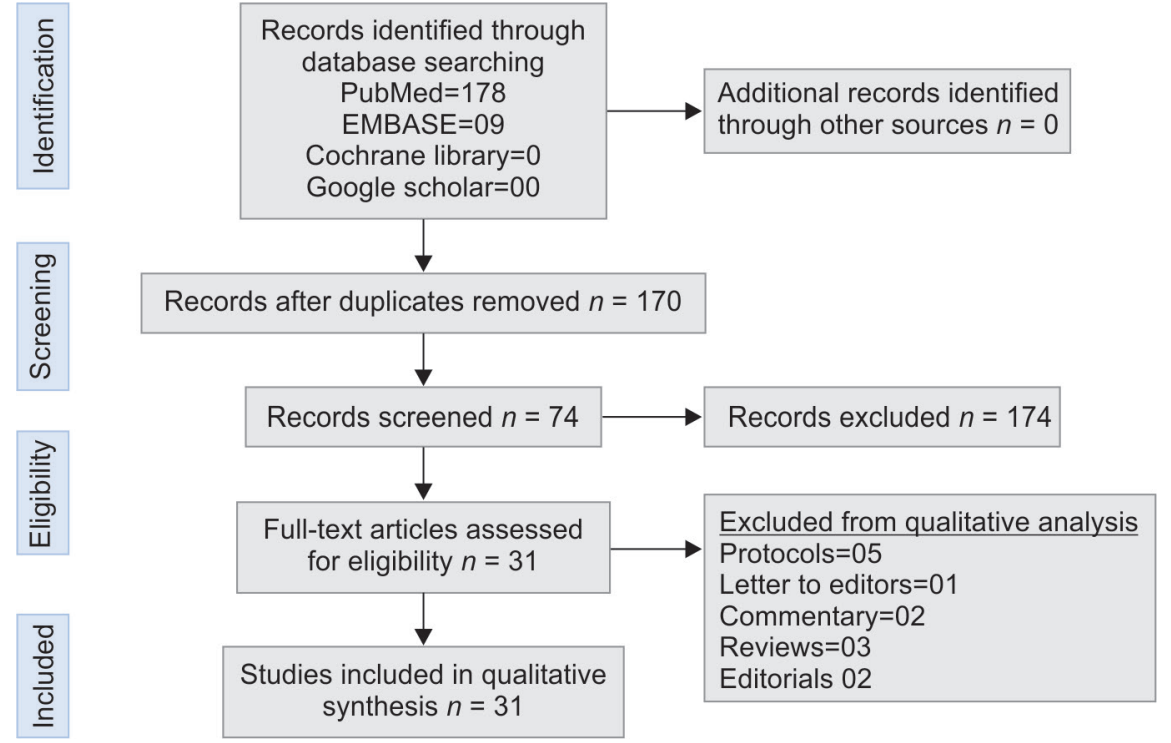




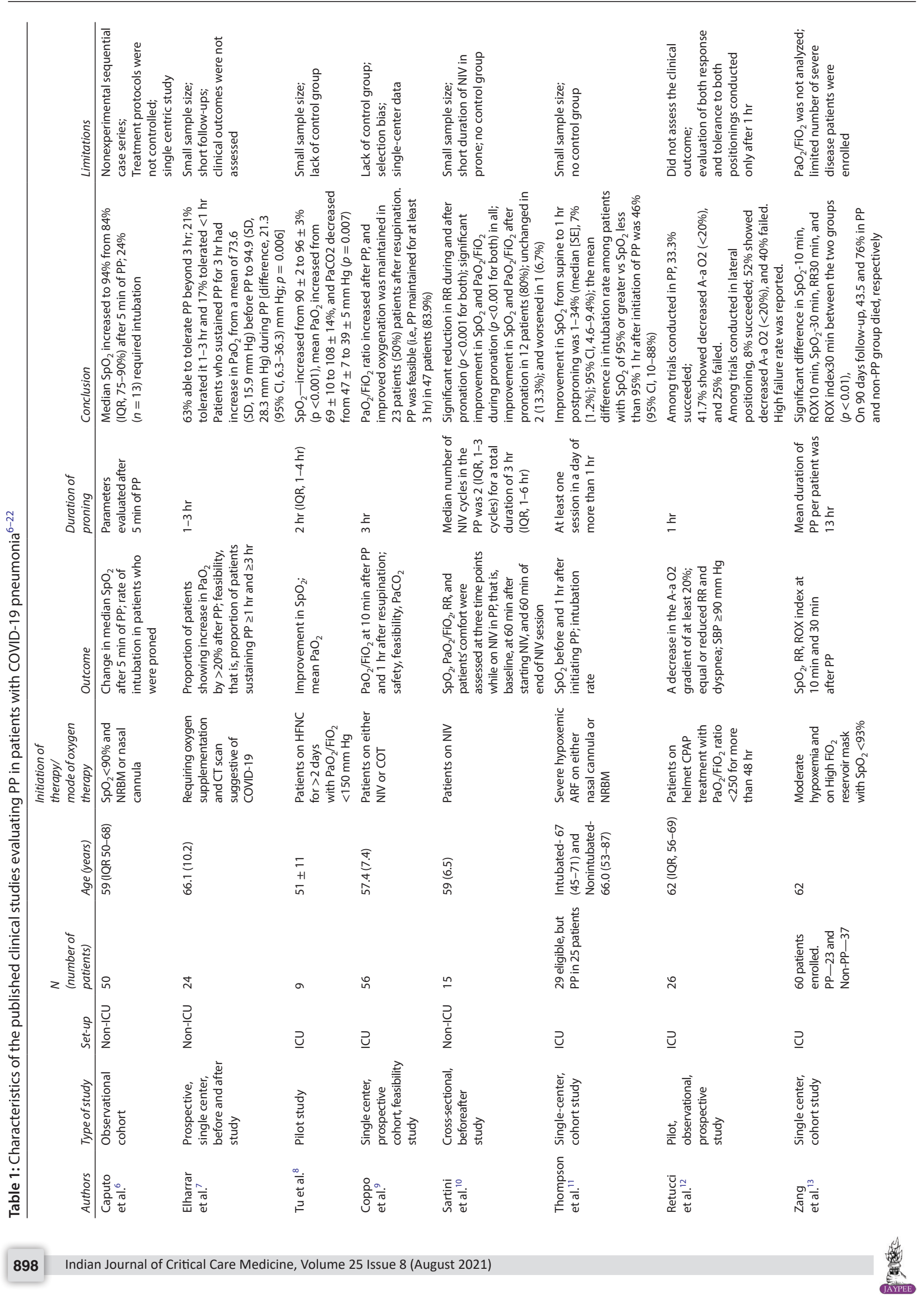




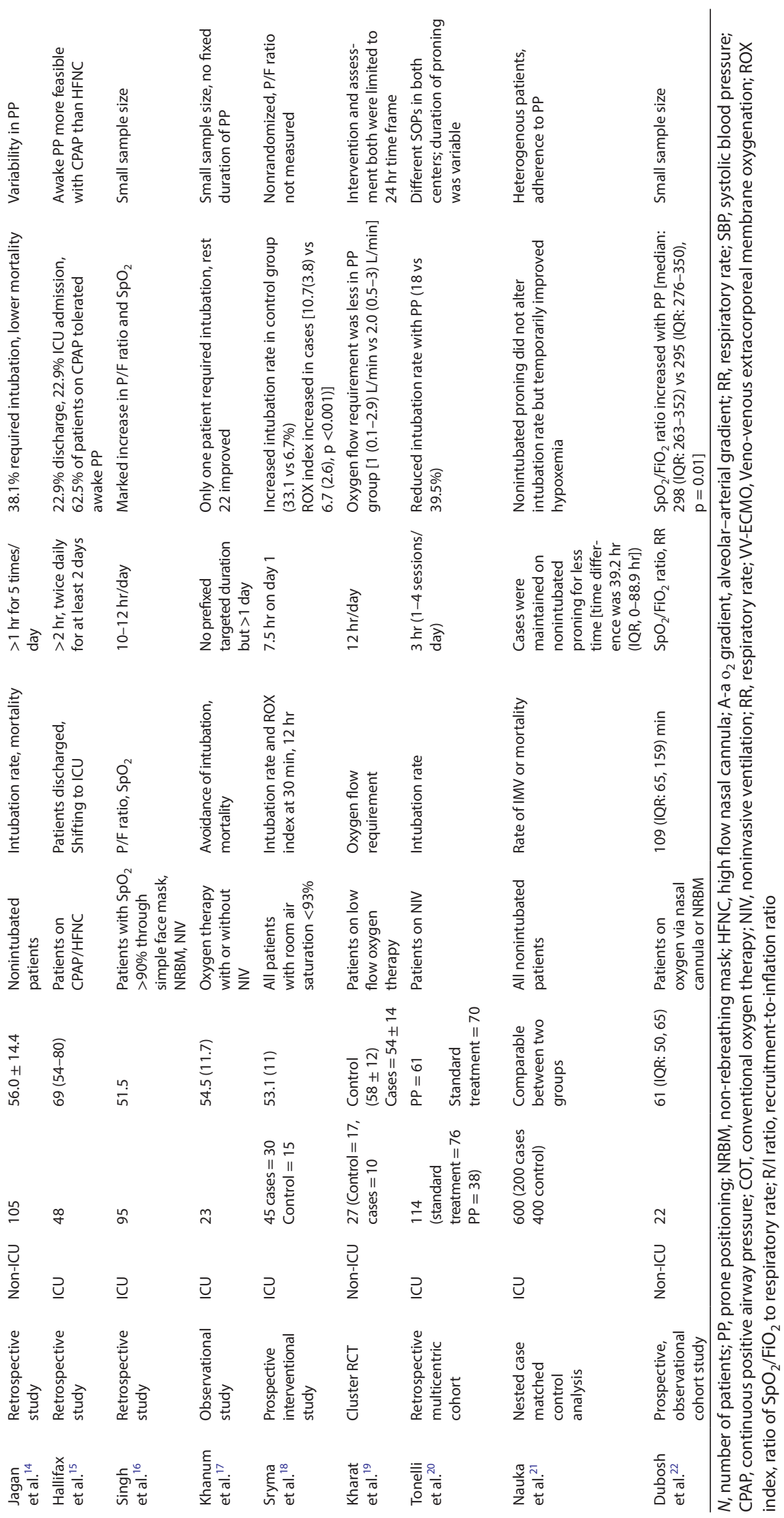


$\mathrm{PP}$, the range of improvement in $\mathrm{SpO}_{2}$ was from 1 to $34 \%$, and the mean difference in intubation rates between patients with $\mathrm{SpO}_{2}$ higher than $95 \%$ compared to those with less than $95 \%$ at 1 hour after PP was $46 \%$.

Retucci et al. ${ }^{12}$ in an observational study evaluated PP and lateral positioning in 26 patients on helmet continuous positive airway pressure (CPAP) and observed a high failure rate.

Table 2 shows the characteristics of the case series evaluating PP in patients with COVID-19, and all had used awake PP. ${ }^{23-31}$ The mean age of all 69 patients included in the case series was 58.0 years. The mean duration of single-PP session extended from 2 hours till 16 hours/ day. The $\mathrm{PaO}_{2} / \mathrm{FiO}_{2}$ ratio, $\mathrm{SpO}_{2}$, clinical improvement, and oxygen requirements were the most commonly used clinical outcomes.

Four case series involving 43 patients evaluating awake PP in SARS-CoV-2 were conducted in non-ICU areas, ${ }^{24,25,28,29}$ out of which, few were conducted among patients receiving helmet NIV. ${ }^{28-30}$ Ripollo-Gallardo et al. ${ }^{28}$ and Bastoni et al. ${ }^{29}$ in their retrospective series of 13 and 10 patients evaluated PP among patients on helmet NIV in the general ward and Emergency Department, respectively. Both studies showed improved oxygenation with PP; however, the latter in addition conducted lung ultrasonography (USG) and did not observe any change in recruitability with the PP. As far as the feasibility of PP with helmet NIV is concerned, it was reported as $92.3 \%$ by Ripollo-Gallardo et al. ${ }^{28}$ and $60 \%$ by Bastoni et al. ${ }^{29}$

$\mathrm{Ng}$ et al. ${ }^{25}$ evaluated PP in 10 patients in the general ward with the $\mathrm{FiO}_{2}$ requirement $<0.5$; three patients were later shifted to ICU where one died. The rest seven patients showed improvement in clinical symptoms. No $\mathrm{PaO}_{2} / \mathrm{FiO}_{2}$ ratio could be assessed due to the setting of the study. Out of five case series that were conducted in the ICU setup, three were conducted among patients receiving HFNC, ${ }^{23,26,27}$ one with helmet $\mathrm{CPAP}^{30}{ }^{30}$ and one on non-rebreathing mask (NRBM). ${ }^{31}$

Table 3 shows the details of various case reports in this context. ${ }^{32-41}$ The mean duration of PP in these case reports ranged from 1 hour till 16-18 hours/day (Table 3).

\section{Specific Subpopulation}

\section{Awake PP in ICU and Non-ICU Setups}

Out of all 1,385 participants, $78.9 \%(n=1,093)$ received awake PP in ICU and $21.0 \%(n=292)$ received it in non-ICU areas, including emergency areas, wards, etc.

\section{Awake PP in Pregnant Patient and Morbidly Obese}

There has been little evidence regarding the use of PP in pregnant women with COVID-19. The only reported use of PP in pregnant patients with COVID-19 was found to be efficacious when combined with HFNC. However, the practical applicability or use of PP in the pregnant patient was a concern. ${ }^{28}$ Recently, Paul et al. reported a morbidly obese (body mass index, $65 \mathrm{~kg} / \mathrm{m}^{2}$ ) COVID-19 patient with obstructive sleep apnea and reported notable improvement in $\mathrm{FiO}_{2}$ requirements titrated down to 0.4 within 1 hour of proning, which persisted even on return to supine position. ${ }^{29}$

\section{Awake PP along with HFNC}

Nine articles that include two clinical studies, 8,15 three case series, ${ }^{23,26,27}$ and five case reports ${ }^{34,36-39}$ including a total of 90 patients have evaluated awake PP along with HFNC in the management of COVID-19 pneumonia. This combination was found to be feasible, helpful, and efficacious in terms of various oxygenation outcomes, for example, $\mathrm{PaO}_{2} / \mathrm{FiO}_{2}, \mathrm{RR}, \mathrm{SpO}_{2}$, and other clinical parameters, and also in a single case report on a pregnant patient with COVID-19 pneumonia. ${ }^{36}$ However, there has been a lack of well-designed studies to validate this finding.

\section{Awake PP with Helmet CPAP}

Only one clinical study ${ }^{12}$ and three case series ${ }^{28-30+}$ including 59 patients have evaluated PP along with helmet CPAP. Retucci et al. observed a high failure rate with PP and lateral positioning in patients receiving helmet CPAP. ${ }^{12}$ On the contrary, all the three case series found PP with helmet CPAP to be feasible and efficacious ${ }^{28-30}$ however, the sustained improvement in oxygenation even after 12 hours of PP was documented only by Golestani et al. ${ }^{30}$

\section{Risk of Bias (Quality) Assessment}

In order to assess the risk of bias of the included studies, the Cochrane Collaboration tool, namely ROBINS-I ("Risk of Bias In Non-randomized Studies-of Interventions"), was used. It is a tool for evaluating the risk of bias from nonrandomized studies utilizing interventions. ${ }^{61}$ The ROBINS-I assesses the risk of bias in seven domains: (1) bias due to confounding, (2) bias due to selection of participants, (3) bias in classification of interventions, (4) bias due to deviation from intended intervention, (5) bias due to missing data, (6) bias in the measurement of outcomes, and (7) bias in the selection of the reported result. Each aforementioned parameter of bias in each study will be scored as having low, medium, high, or unclear risk. The study with lower risk is deemed as a high-quality study. The risk of bias was independently assessed by GCT and ZA, and disagreements were resolved through discussion with $\mathrm{MM}$ The overall judgment on the bias assessment following assessment of each domain of the included studies in the present systematic review as per the ROBINS-I tool has been found to have moderate to serious risk. ${ }^{61}$ The risk of bias was variable among different included studies. The weighted summary plot of different aforementioned biases among all nonrandomized studies ${ }^{6-22}$ was designed using robvis web app (Fig. 1). ${ }^{62}$

\section{Discussion}

The present review has summarized the current evidence of awake PP in patients with SARS-CoV-2, out of a total of 1,385 patients in whom awake PP was evaluated. Overall, the technique was found to be efficacious in terms of improvement in oxygenation in $78.9 \%$ $(n=1,093)$ of patients in ICU and $21.0 \%(n=292)$ in non-ICU areas, including emergency areas, wards, etc. Awake PP along with HFNC was used in 90 patients and was found to be efficacious in all the cases. Awake PP was used in patients with helmet CPAP in 59 cases with inconclusive results. However, none of the studies have evaluated the optimal duration of awake PP; in the majority, the oxygenation parameters were evaluated within few minutes to only a few hours after PP, and no long-term outcomes were assessed.

PP reverses the compression atelectasis of the dorsal lungs due to heart and mediastinum and helps alveolar recruitment in the dorsal lung (now nondependent) by increasing the transpulmonary pressure leading to the homogenous distribution of ventilation across the lung. ${ }^{2,3}$ However, perfusion remains higher in the dorsal region due to higher production of nitric oxide (a potent vasodilator) in the endothelium of the dorsal lung leading to a 


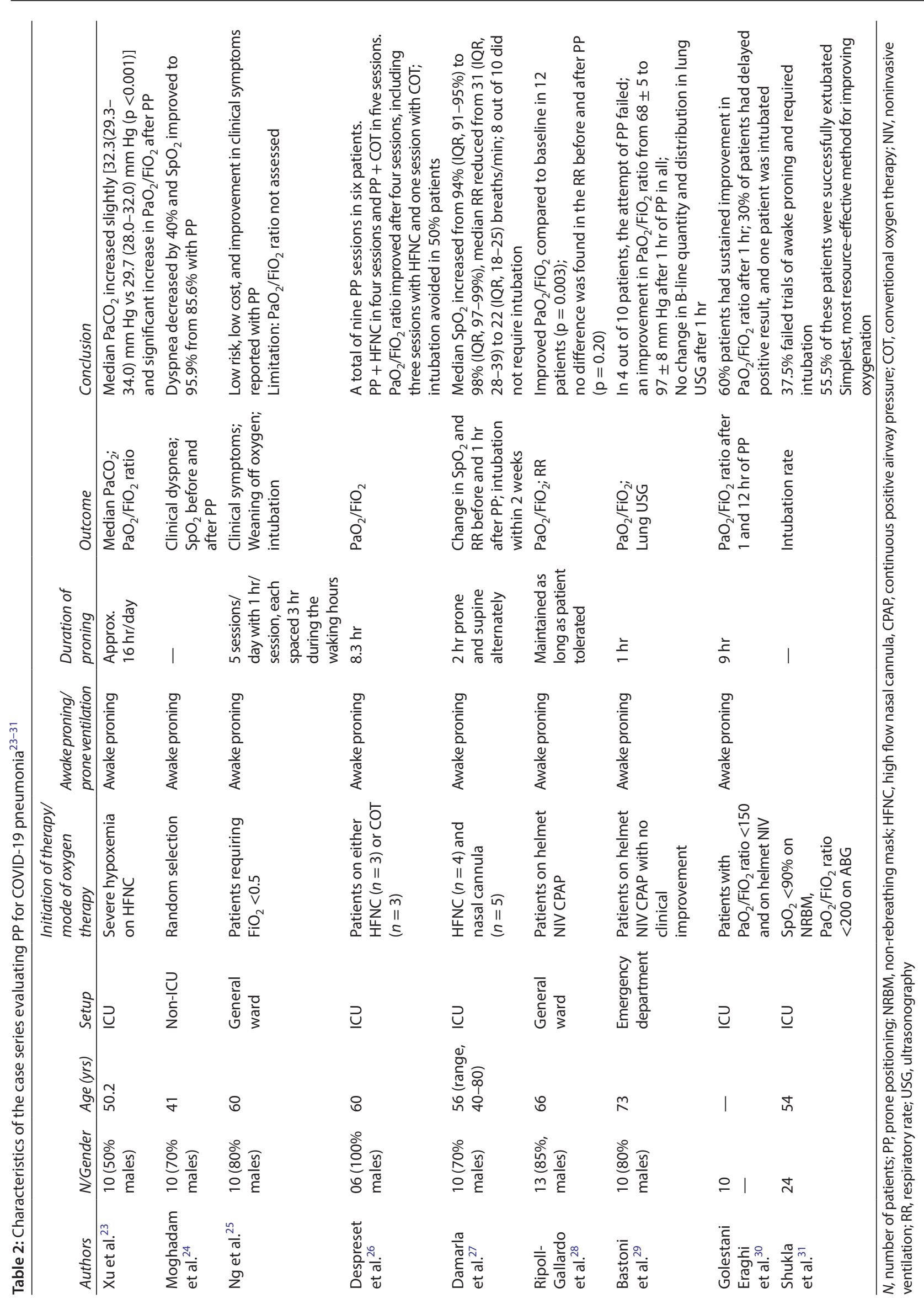




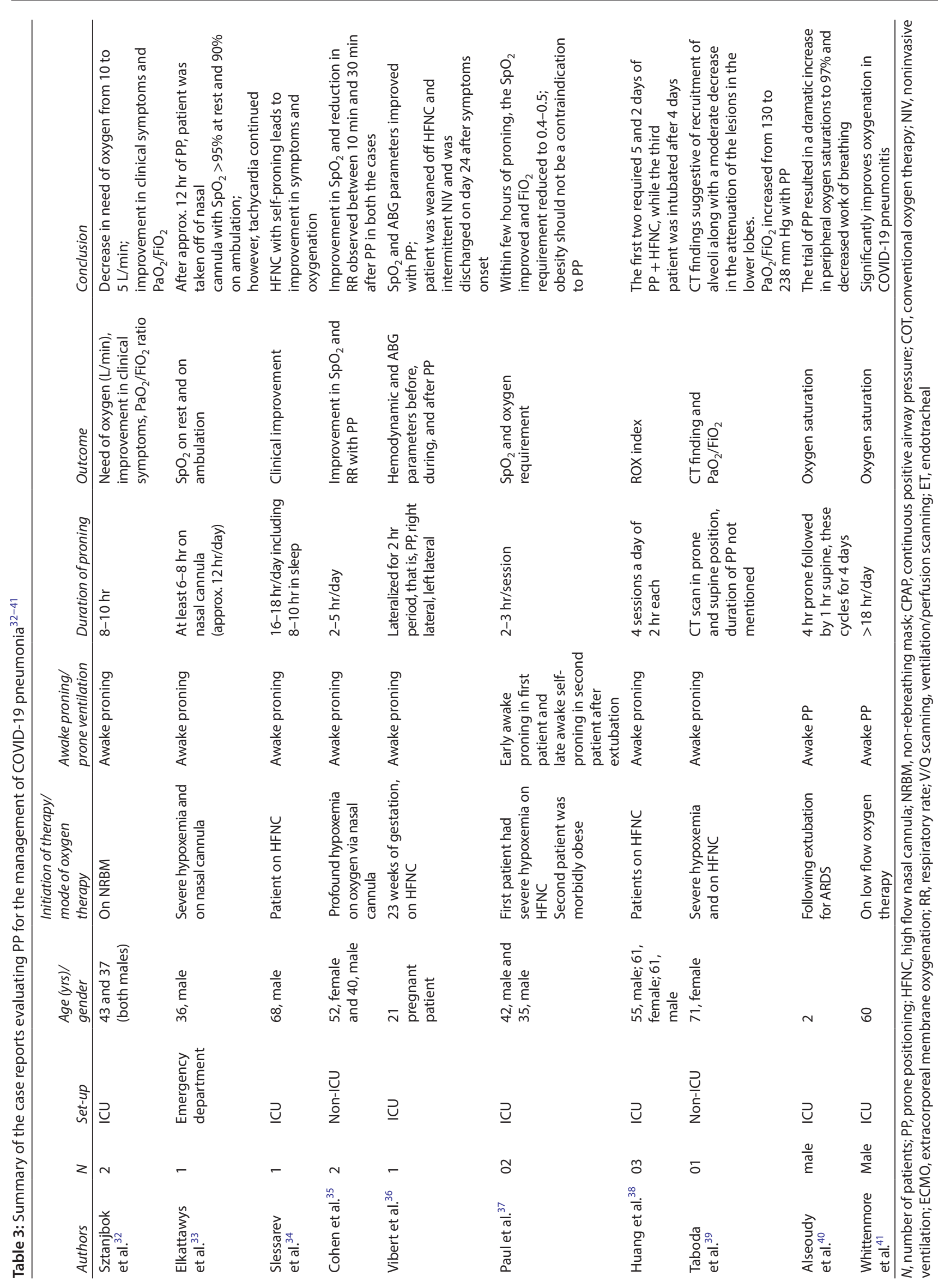


Bias due to confounding

Bias due to selection of participants

Bias in classification of interventions

Bias due to deviations from intended interventions

Bias due to missing data

Bias in measurement of outcomes

Bias in selection of the reported result

Overall risk of bias

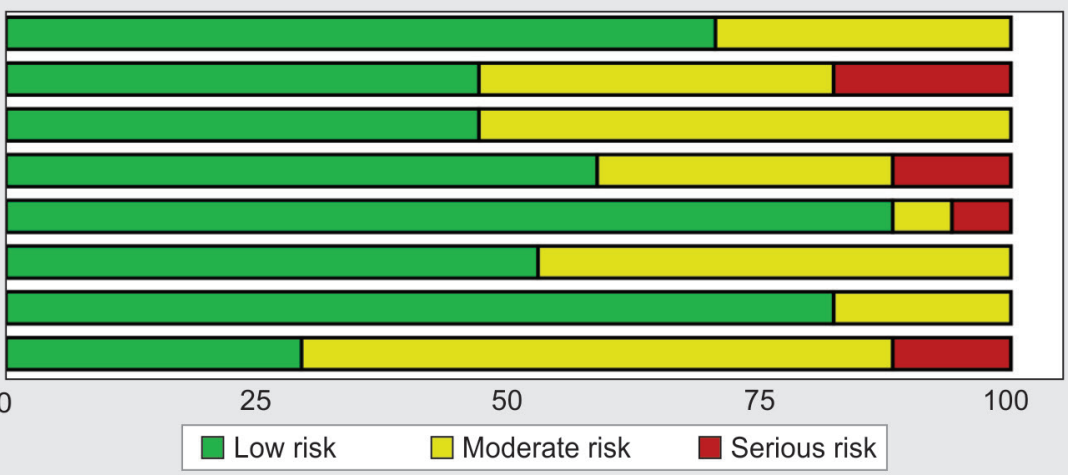

Fig. 1: Risk of bias assessment using ROBINS-I tool ${ }^{62}$

significant reduction in the relative shunt fraction along with the improvement in $\mathrm{PaO}_{2} / \mathrm{FiO}_{2}$. The role of prone ventilation in intubated ARDS patients has been established since the last few years. ${ }^{2,3}$ Extrapolating the evidence of PP in mechanically ventilated non-COVID ARDS patients, recently, even the Surviving Sepsis Campaign panel has advocated a trial of PP in mechanically ventilated COVID-19 patients with moderate to severe ARDS for a period of 12-16 hours. ${ }^{63}$

In the present COVID-19 pandemic, the use of PP has been widely extended to awake patients in both ICU and non-ICU areas. Awake PP is an important adjuvant for improving oxygenation in ARDS along with other factors like $\mathrm{FiO}_{2}$ delivered, mode of oxygen therapy, and other treatment protocols. HFNC has been known to increase the ventilator-free days and decrease the mortality in ICU patients. In the present systematic review, we observed a striking improvement in oxygenation with PP in patients who were receiving HFNC. We are also looking forward to the results of the ongoing metatrial to investigate awake PP in COVID-19 patients undergoing HFNC. ${ }^{45}$ However, with helmet NIV, its efficacy has been found to be contentious.

There have been concrete evidences regarding the optimal duration of prone ventilation in non-COVID ARDS ${ }^{2,3}$; however, no study has assessed the optimal duration of awake PP since its inception has gained momentum in COVID-19 pandemic. The majority of the clinical studies evaluating awake PP in COVID-19 pneumonia have assessed the oxygenation as early as 5 minutes to the maximum of 1 to 3 hours after PP. ${ }^{6,9,11-13}$ The sustained improvement in oxygenation as assessed in a single study by Golestani et al. after 12 hours was observed in only $30 \%$ of patients. ${ }^{30}$ In addition, only a few studies have utilized control group for the evaluation of awake PP. ${ }^{13,18,19}$ In all these studies, the assessment for improved oxygenation was undertaken as early as 1 hour ${ }^{13,18}$ till the end of day one. ${ }^{19}$ These studies' results highlighting the improvement in oxygenation following such a short duration of awake PP could possibly be implicated to the transient lung recruitment. ${ }^{5}$ This mandates the need to determine the optimal duration of awake PP along with the assessment of long-term clinical outcomes, for example, intubation rates and mortality.

The present systematic literature review is dealt with few limitations. Firstly, the studies included had small sample sizes and single centric data.
Secondly, except for one, ${ }^{13}$ all studies lacked the control group. Thirdly, considering the fact that early use of awake PP may improve the overall prognosis, the evidence regarding the efficacy and safety of the institution of PP in outside ICU setups has been found to be insufficient. Interestingly, a clinical study by Sartini et al. ${ }^{10}$ and two case series ${ }^{28,29}$ have evaluated PP in severely hypoxemic patients receiving NIV and helmet CPAP in non-ICU areas, a finding which cannot be related in developing countries with limited facilities during this pandemic times. Fourthly, there was a heterogeneous patient population in terms of the mode of oxygen therapy or respiratory support, duration of proning, treatment protocol, and severity of illness at the time of initiation of PP. Also, few studies displayed incomplete data, particularly the mode of oxygen therapy and duration of proning.

In conclusion, the literature available so far encourages the use of early awake self-proning in addition to its use in intubated patients in the management of SARS-CoV-2 infection. The shortterm improvement in the oxygenation as reported in various trials could simply be a "recruitment maneuver" as the majority of the oxygenation outcome parameters were assessed between few minutes to 3 hours after PP in various studies. The overall evidence pertaining to the use of awake proning in the management of COVID-19 disease is not sufficient as there has been a lack of randomized controlled trial, and therefore, further well-designed multicentric studies with larger sample size and preferably with a control group are warranted to evaluate the PP as an adjunct in the management of COVID-19 pneumonia in terms of its safety, optimal duration of proning, and efficacy in improving oxygenation with individual modes of oxygen therapy.

\section{OrCID}

Geetanjali T Chilkoti @ https://orcid.org/0000-0002-2436-0444 Medha Mohta $\odot$ https://orcid.org/0000-0003-4222-8428

Ashok K Saxena ㄴ) https://orcid.org/0000-0003-1251-9320

Zainab Ahmad (1) https://orcid.org/0000-0002-5944-607X

Chhavi S Sharma @ https://orcid.org/0000-0002-4852-2065

\section{References}

1. Gordon A, Rabold E, Thirumala R, Husain AA, Patel S, Cheema T. Prone positioning in ARDS. Crit Care Nurs Q 2019;42(4):371-375. DOI: 10.1097/CNQ.0000000000000277. 
2. Munshi L, Del Sorbo L, Adhikari NKJ, Hodgson CL, Wunsch $H$, Meade $\mathrm{MO}$, et al. Prone position for acute respiratory distress syndrome. A systematic review and meta-analysis. Ann Am Thorac Soc 2017;14(4):S280-S288. DOI: 10.1513/AnnalsATS.201704-343OT.

3. Bloomfield R, Noble D, Sudlow A. Prone position for acute respiratory failure in adults. Cochrane Database Syst Rev 2015;11:CD008095. DOI: 10.1002/14651858.CD008095.pub2.

4. Bamford P, Bentley A, Dean J, Whitmore D. ICS guidance for prone positioning of the conscious COVID patient. 2020.

5. Chad T, Sampson C. Prone positioning in conscious patients on medical wards: a review of the evidence and its relevance to patients with COVID-19 infection. Clin Med (Lond) 2020;20(4):e97-e103. DOI: 10.7861/clinmed.2020-0179.

6. Caputo ND, Strayer RJ, Levitan R. Early self-proning in awake, non-intubated patients in the emergency department: a single ED's experience during the covid-19 pandemic. Acad Emerg Med 2020;27(5):375-378. DOI: 10.1111/acem.13994.

7. Elharrar X, Trigui Y, Dols AM, Touchon F, Martinez S, Prud'homme E, et al. Use of prone positioning in nonintubated patients with COVID-19 and hypoxemic acute respiratory failure. JAMA 2020;323(22):2336-2338. DOI: 10.1001/jama.2020.8255.

8. Tu GW, Liao YX, Li QY, Dong H, Yang LY, Zhang XY, et al. Prone positioning in high-flow nasal cannula for COVID-19 patients with severe hypoxemia: a pilot study. Ann TransI Med 2020;8(9):598. DOI: 10.21037/atm-20-3005.

9. Coppo A, Bellani G, Winterton D, Di Pierro M, Soria A, Faverio P, et al. Feasibility and physiological effects of prone positioning in nonintubated patients with acute respiratory failure due to COVID-19 (PRON-COVID): a prospective cohort study. Lancet Respir Med 2020;8(8):765-774. DOI: 10.1016/S2213-2600(20)30268-X.

10. Sartini C, Tresoldi M, Scarpellini P, Tettamanti A, Carcò F, Landoni G, et al. Respiratory parameters in patients with COVID-19 after using noninvasive ventilation in the prone position outside the intensive care unit. JAMA 2020;323(22):2338-2340. DOI: 10.1001/jama.2020.7861.

11. Thompson $A E$, Ranard $B L$, Wei $Y$, Jelic S. Prone positioning in awake, nonintubated patients with COVID-19 Hypoxemic respiratory failure. JAMA Intern Med 2020;e203030. DOI: 10.1001/ jamainternmed.2020.3030.

12. Retucci M, Aliberti S, Ceruti C, Santambrogio M, Tammaro S, Cuccarini F, et al. Prone and lateral positioning in spontaneously breathing patients with COVID-19 pneumonia undergoing noninvasive helmet CPAP treatment. Chest 2020:S0012-3692(20)31888-2. DOI: 10.1016/ j.chest.2020.07.006.

13. Zang X, Wang Q, Zhou H, Liu S, Xue X; COVID-19 Early Prone Position Study Group. Efficacy of early prone position for COVID-19 patients with severe hypoxia: a single-center prospective cohort study. Intensive Care Med 2020;46(10):1-3. DOI: 10.1007/s00134-020-06182-4.

14. Jagan N, Morrow LE, Walters RW, Klein LP, Wallen TJ, Chung J, et al. The POSITIONED study: prone positioning in nonventilated coronavirus disease 2019 patients-a retrospective analysis. Crit Care Explor 2020;2(10):e0229. DOI: 10.1097/CCE.0000000000000229.

15. Hallifax RJ, Porter BM, Elder PJ, Evans SB, Turnbull CD, Hynes G, et al. Successful awake proning is associated with improved clinical outcomes in patients with COVID-19: single-centre high-dependency unit experience. BMJ Open Respir Res 2020;7(1):e000678. DOI: 10.1136/bmjresp-2020-000678.

16. Singh $\mathrm{P}$, Jain $\mathrm{P}$, Deewan H. Awake prone positioning in COVID-19 patients. Indian J Crit Care Med 2020;24(10):914-918. DOI: 10.5005/ jp-journals-10071-23546.

17. Khanum I, Samar F, Fatimah Y, Safia A, Adil A, Kiren H, et al. Role of awake prone positioning in patients with moderate-to-severe COVID-19: an experience from a developing country. Monaldi Arch Chest Dis 2021;91(2):10. DOI: 10.4081/monaldi.2021.1561.

18. Sryma PB, Mittal S, Mohan A, Madan K, Tiwari P, Bhatnagar S, et al. Effect of proning in patients with COVID-19 acute hypoxemic respiratory failure receiving noninvasive oxygen therapy. Lung India 2021;38(Suppl.):S6-S10. DOI: 10.4103/lungindia.lungindia_794_20.
19. Kharat A, Dupuis-Lozeron E, Cantero C, Marti C, Grosgurin O, Lolachi S, et al. Self-proning in COVID-19 patients on low-flow oxygen therapy: a cluster randomised controlled trial. ERJ Open Res 2021;7(1):006922020. DOI: 10.1183/23120541.00692-2020.

20. Tonelli R, Pisani L, Tabbì L, Comellini V, Prediletto I, Fantini R, et al. Early awake proning in critical and severe COVID-19 patients undergoing noninvasive respiratory support: a retrospective multicenter cohort study [published online ahead of print, $2021 \mathrm{Mar} 22$ ]. Pulmonology 2021;S2531-0437(21)00077-5. DOI: 10.1016/j.pulmoe.2021.03.002.

21. Nauka PC, Chekuri S, Aboodi M, Hope AA, Gong MN, Chen JT. A case-control study of prone positioning in awake and nonintubated hospitalized coronavirus disease 2019 patients. Crit Care Explor 2021;3(2):e0348. DOI: 10.1097/CCE.0000000000000348.

22. Dubosh NM, Wong ML, Grossestreuer AV, Loo YK, Sanchez LD, Chiu D, et al. Early, awake proning in emergency department patients with COVID-19. Am J Emerg Med 2020:S0735-6757(20)31105-0. DOI: 10.1016/j.ajem.2020.11.074.

23. Xu Q, Wang T, Qin X, Jie Y, Zha L, Lu W. Early awake prone position combined with high-flow nasal oxygen therapy in severe COVID-19: a case series. Crit Care 2020;24(1):250. DOI: 10.1186/s13054-020-02991-7.

24. Moghadam VD, Shafiee H, Ghorbani M, Heidarifar R. Prone positioning in management of COVID-19 hospitalized patients. Rev Brasil Anestesiol 2020;70(2):188-190. DOI: 10.1016/j.bjan.2020.05.001.

25. Ng Z, Tay WC, Ho CHB. Awake prone positioning for non-intubated oxygen dependent COVID-19 pneumonia patients. Eur Respir J 2020;56(1):2001198. DOI: 10.1183/13993003.01198-2020.

26. Despres C, Brunin Y, Berthier F, Pili-Floury S, Besch G. Prone positioning combined with high-flow nasal or conventional oxygen therapy in severe Covid-19 patients. Crit Care 2020;24(1):256. DOI: 10.1186/s13054-020-03001-6.

27. Damarla M, Zaeh S, Niedermeyer S, Merck S, Niranjan-Azadi A, Broderick $B$, et al. Prone positioning of nonintubated patients with COVID-19. Am J Respir Crit Care Med 2020;202(4):604-606. DOI: 10.1164/rccm.202004-1331LE.

28. Ripoll-Gallardo A, Grillenzoni L, Bollon J, Della Corte F, Barone-Adesi F. Prone positioning in non-intubated patients with COVID-19 outside of the intensive care unit: more evidence needed. Disaster Med Public Health Prep 2020;1-3. DOI: 10.1017/dmp.2020.267.

29. Bastoni D, Poggiali E, Vercelli A, Demichele E, Tinelli V, lannicelli T, et al. Prone positioning in patients treated with non-invasive ventilation for COVID-19 pneumonia in an Italian emergency department. Emerg Med J 2020;37(9):565-566. DOI: 10.1136/ emermed-2020-209744.

30. Golestani-Eraghi M, Mahmoodpoor A. Early application of prone position for management of Covid-19 patients [published online ahead of print, 2020 May 26]. J Clin Anesth 2020;66:109917. DOI: 10.1016/j.jclinane.2020.109917.

31. Shukla U, Chavali S, Mukta P, Mapari A, Vyas A. Initial experience of critically ill patients with COVID-119 in Western India: a case series. Indian J Crit Care Med 2020;24(7):509-513. DOI: 10.5005/ jp-journals-10071-23477.

32. Sztajnbok J, Maselli-Schoueri JH, Cunha de Resende Brasil LM, Farias de Sousa L, Cordeiro CM, Sansão Borges LM, et al. Prone positioning to improve oxygenation and relieve respiratory symptoms in awake, spontaneously breathing non-intubated patients with COVID-19 pneumonia. Respir Med Case Rep 2020;30:101096. DOI: 10.1016/j. rmcr.2020.101096.

33. Elkattawy S, Noori M. A case of improved oxygenation in SARS-CoV-2 positive patient on nasal cannula undergoing prone positioning. Respir Med Case Rep 2020;30:101070. DOI: 10.1016/j.rmcr.2020.101070.

34. Slessarev M, Cheng J, Ondrejicka M, Arntfield R; Critical Care Western Research Group. Patient self-proning with high-flow nasal cannula improves oxygenation in COVID-19 pneumonia. Can J Anaesth 2020;67(9):1288-1290. DOI: 10.1007/s12630-020-01661-0.

35. Cohen D, Wasserstrum Y, Segev A, Avaky C, Negru L, Turpashvili N, et al. Beneficial effect of awake prone position in hypoxaemic patients with COVID-19: case reports and literature review. Intern 
Med J 2020:10.1111/imj.14926. DOI: 10.1111/imj.14926. PMID: 32697030; PMCID: PMC7404489.

36. Vibert F, Kretz M, Thuet V, Barthel F, De Marcillac F, Deruelle P, et al. Prone positioning and high-flow oxygen improved respiratory function in a 25-week pregnant woman with COVID-19. Eur J Obstet Gynecol Reprod Biol 2020;250:257-258. DOI: 10.1016/j.ejogrb.2020.05.022.

37. Paul V, Patel S, Royse M, Odish M, Malhotra A, Koenig S. Proning in nonintubated (PINI) in times of COVID-19: case series and a review. J Intensive Care Med 2020;35(8):818-824. DOI: 10.1177/0885066620934801.

38. Huang CF, Zhuang YF, Liu J, Tay CK, Sewa DW. Rationale and significance of patient selection in awake prone positioning for COVID-19 pneumonia. Eur Respir J 2020;2002173. DOI: 10.1183/13993003.02173-2020.

39. Taboada M, Rodríguez N, Riveiro V, Baluja A, Atanassoff PG. Prone positioning in awake non-ICU patients with ARDS caused by COVID-19. Anaesth Crit Care Pain Med. 2020;39(5):581-583. DOI:10.1016/j.accpm.2020.08.002.

40. Als eoudy MM, Abo Elfetoh MA, Alrefaey AK. Awake proning of a 2-year-old extubated child with severe COVID-19 pneumonitis. Anaesth Rep 2020;8(2):183-186. DOI: 10.1002/anr3.12084.

41. Whittemore P, Macfarlane L, Herbert A, Farrant J. Use of awake proning to avoid invasive ventilation in a patient with severe COVID-19 pneumonitis. BMJ Case Rep 2020;13(8):e236586. DOI: 10.1136/bcr-2020-236586.

42. Jiang LG, LeBaron J, Bodnar D, Caputo ND, Chang BP, Chiricolo G, et al. Conscious proning: an introduction of a proning protocol for nonintubated, awake, hypoxic emergency department COVID-19 patients. Acad Emerg Med 2020;27(7):566-569. DOI: 10.1111/acem.14035.

43. Bower $\mathrm{G}, \mathrm{He} \mathrm{H}$. Protocol for awake prone positioning in COVID-19 patients: to do it earlier, easier, and longer. Crit Care 2020;24(1):371. DOI: 10.1186/s13054-020-03096-x.

44. Longhini F, Bruni A, Garofalo E, Navalesi P, Grasselli G, Cosentini R, et al. Helmet continuous positive airway pressure and prone positioning: A proposal for an early management of COVID-19 patients. Pulmonology 2020;26(4):186-191. DOI: 10.1016/j.pulmoe. 2020.04.014.

45. Li J, Pavlov I, Laffey JG, Roca O, Mirza S, Perez Y, et al. Meta-trial of awake prone positioning with nasal high flow therapy: Invitation to join a pandemic collaborative research effort. J Crit Care 2020;60:140 142. DOI: 10.1016/j.jcrc.2020.07.020.

46. Bentley SK, lavicoli L, Cherkas D, Lane R, Wang E, Atienza M, et al. Guidance and patient instructions for proning and repositioning of awake, nonintubated COVID-19 patients. Acad Emerg Med 2020:10.1111/acem.14067. DOI: 10.1111/acem.14067. PMID: 32597005; PMCID: PMC7361422.

47. Nasa P, Azoulay E, Khanna AK, et al. Expert consensus statements for the management of COVID-19-related acute respiratory failure using a Delphi method. Crit Care 2021;25(1):106. DOI: 10.1186/ s13054-021-03491-y.

48. Stilma W, Åkerman $\mathrm{E}$, Artigas $\mathrm{A}$, et al. Awake proning as an adjunctive therapy for refractory hypoxemia in non-intubated patients with
COVID-19 acute respiratory failure: guidance from an international group of healthcare workers. Am J Trop Med Hyg 2021;104(5):16761686. DOI: 10.4269/ajtmh.20-1445.

49. LindahI SGE. Using the prone position could help to combat the development of fast hypoxia in some patients with COVID-19. Acta Paediatr 2020;109(8):1539-1544. DOI: 10.1111/apa.15382.

50. Prasad M, Visrodia K. Should I prone non-ventilated awake patients with COVID-19? [published online ahead of print, 2020 Jun 30]. Cleve Clin J Med 2020;10. DOI: 10.3949/ccjm.87a.ccc050.

51. Flynn Makic MB. Prone position of patients with COVID-19 and acute respiratory distress syndrome. J Perianesth Nurs 2020;35(4):437-438. DOI: 10.1016/j.jopan.2020.05.008.

52. Sodhi K, Chanchalani G. Awake proning: current evidence and practical considerations. Indian J Crit Care Med 2020;24(12): 1236-1241. DOI: 10.5005/jp-journals-10071-23684.

53. Sen MK, Gupta N, Ish P, Kumar R, Yadav SR. Awake proning in Covid-19 pneumonia. Infez Med. 2020 Sep 1;28(3):453-455. PMID: 32920584.

54. Raoof S, Nava S, Carpati C, Hill NS. High-flow, noninvasive ventilation and awake (nonintubation) proning in patients with coronavirus disease 2019 with respiratory failure. Chest 2020;158(5):1992-2002. DOI: 10.1016/j.chest.2020.07.013.

55. Khan S, Choudry E, Mahmood SU, Mulla AY, Mehwish S. Awake proning: a necessary evil during the COVID-19 pandemic. Cureus 2020;12(7):e8989. DOI: 10.7759/cureus.8989.

56. Ghelichkhani P, Esmaeili M. Prone Position in Management of COVID-19 Patients; a Commentary. Arch Acad Emerg Med. 2020 Apr 11;8(1):e48. PMID: 32309812; PMCID: PMC7158870.

57. McNicholas B, Cosgrave D, Giacomini C, Brennan A, Laffey JG. Prone positioning in COVID-19 acute respiratory failure: just do it? Br J Anaesth 2020;50007-0912(20)30443-8. DOI: 10.1016/ j.bja.2020.06.003.

58. Munshi L, FralickM, Fan E. Prone positioning in non-intubated patients with COVID-19: raising the bar. Lancet Respir Med 2020;8(8):744-745. DOI: 10.1016/S2213-2600(20)30269-1.

59. Telias I, Katira BH, Brochard L. Is the prone position helpful during spontaneous breathing in patients with COVID-19? JAMA 2020;323(22):2265-2267. DOI: 10.1001/jama.2020.8539.

60. Garg R. Conscious proning or mixed positioning for improving oxygenation-COVID-19 brings many changes! Indian J Crit Care Med 2020;24(10):893-894. DOI: 10.5005/jp-journals-10071-23624.

61. Sterne JA, Hernán MA, Reeves BC, Savović J, Berkman ND, Viswanathan M, et al. ROBINS-I: a tool for assessing risk of bias in non-randomised studies of interventions. BMJ 2016;355:i4919. DOI: 10.1136/bmj.i4919.

62. McGuiness LA. robvis: An R Package and web application for visualizing risk-of-bias assessments. 2019. Retrieved from: https:// github.com/mcguinlu/robvis [Accessed December 15, 2020].

63. Alhazzani W, Møller MH, Arabi YM, Loeb M, Gong MN, Fan E, et al. Surviving sepsis campaign: guidelines on the management of critically ill adults with coronavirus disease 2019 (COVID-19). Intensive Care Med 2020;46(5):854-887. DOI: 10.1007/s00134-020-06022-5. 